\title{
Transverse-Torsional Vibrations of the High-Speed Planetary Gear Transmission
}

\author{
He Zhaoxia ${ }^{*}, 1,2$, Liu Qingtao ${ }^{1}$, Chang Lehao ${ }^{1}$, Wu Jinsong ${ }^{2}$ \\ ${ }^{I}$ Key Laboratory of Road Construction Technology and Equipment, Ministry of Education, Chang'an University, Xi'an, \\ 710064, China \\ ${ }^{2}$ Jiangsu Huatong Kinetics Co., Ltd., Zhen Jiang, 212003, China
}

\begin{abstract}
The vibration characteristics of a high-speed planetary gear transmission (PGT) are studied in this paper. A transverse-torsional coupling dynamic model is developed using lumped parameter method. In order to ensure the accuracy of the analysis model, the mesh stiffness, support stiffness and the influence of eccentric masses on system are also considered. By solving the eigenvalue of differential equations of motion, the natural frequencies and mode shapes are calculated. According to the vibration modes of this gear transmission, the characteristics of translational mode and torsional mode are described in details. The influence of mesh stiffness, support stiffness and modal modes are discussed by adopting strain and kinetic energy.
\end{abstract}

Keywords: Mesh stiffness, Modal analysis, Strain energy, Planetary gear transmission, Vibration characteristics.

\section{INTRODUCTION}

Planetary gear transmission (PGT) is widely used in the automobiles, helicopters and marine applications. PGT is more advanced than counter-shaft drive because of its higher torque-to-weight ratio, compactness and reduced noise. Despite the distinguishing advantages, how to reduce the noise and vibration of planetary gear transmission remains to be key concern in these applications. Accurate dynamics model is the first work to acquire and improve performance of transmission [1].

The free vibration characterization can be predicted by studying the eigenvalue problems for various lumped parameter models [2]. Lin and Parker developed an analytical model which admitted a three degrees of freedom for each of the sun, ring, carrier and planets [3, 4]. Ambarisha [5] established a non-linear dynamics model of planetary gear system by coupling lumped parameter method and FEM-Contact model which considered backlash in circular tooth. The vibration modes of PGT with helical gears were also simulated using a three-dimensional dynamic model including all six rigid body motions of the carrier and the gears $[6,7]$. According to A. Fernandez del Rincon [8], the deformation at each gear contact point is formulated as a combination of a global and a local term. The former was obtained by means of a finite element model and the latter was described by an analytical approach which is derived from Hertzian contact theory. Z. Yang built a nonlinear torsional dynamic model and performed analysis about the influences of different design parameters [9].

*Address correspondence to this author at the Key Laboratory of Road Construction Technology and Equipment, Ministry of Education, Chang'an University, Nan Er Huan Zhong Duan, Xi'an, Postcard: 710064, China;

Tel: 13649275406; E-mail: hebeier@eyou.com
M. Rashidi built a linear dynamic model to search the effects of shaft location and mesh stiffness on the gear mesh forces [10]. Lehao Chang and Geng Liu analyzed influence of key parameters on vibration of planetary gear system, and these parameters included mesh stiffness, structure stiffness, moment of inertia and mass of structure [11].

In this paper, a transverse-torsional coupling dynamic model for a planetary gear transmission is established. And the mesh stiffness, support stiffness and the eccentric masses are considered in this model. The free vibration characteristics of the PGT are investigated in the following analysis. Then the formulations to acquire the modal kinetic energy, support strain energy and mesh strain energy for different vibration modes are derived.

\section{DYNAMIC MODELING}

A 2K-H PGT to be investigated is used in an aircraft engine, it is made of sun wheel, carrier, ring gear and four planets. And its structure is shown in Fig. (1). The sun S transfers the input torque to the planets $\mathrm{P}$. Then the torque is outputted by the carrier $\mathrm{C}$. And $\mathrm{R}$ is the ring gear of the PGT. The lumped parameter model of the system is shown in Fig. (2). In this system, main components are considered to be rigid and the gear tooth flexibilities are modeled as linear springs on the meshing action plane. The deflections of carrier, sun, ring and planets are described by local rotational and translational coordinates. The axial deflection is not considered due to spur-gear.

$O X Y$ is the moving coordinate system of the center gear, and its origin locates in the theoretical center of the wheel. $O_{i} X_{i} Y_{i}(i=1,2, \cdots, N, N$ is the number of the planets $)$ is the local moving coordinate of planet, and its origin $O_{i}$ coincides with the center of planet $i$. The transitional and torsional displacement of each parts are represented by $x_{j}, y_{j}$ and 


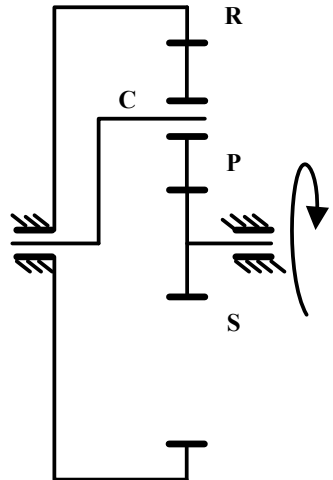

Fig. (1). A 2K-H PGT structure.

$\theta_{j}(j=s, r, c, p i, \ldots, p N)$, which occurred due to vibration of the system. And $E_{j}$ means the eccentric distance. For each gear pair, the translational gear mesh displacements $u_{j}=R_{j} \theta_{j}$ can be replaced by $\theta_{j}$, where $R_{j}$ is the base circle radius for each gear or the assembly radius of the planets for the carrier. According the dynamics model in Fig. (2), the mesh stiffness between the sun and the planet is described by spring element $k_{s p}$, and the mesh stiffness between the ring gear and the planet is denoted by $k_{r p}$. The support stiffness and torsional stiffness are represented by $k_{j x}, k_{j y}$ and $k_{j \mathrm{u}}(j=s$, $r, c, p)$ respectively.

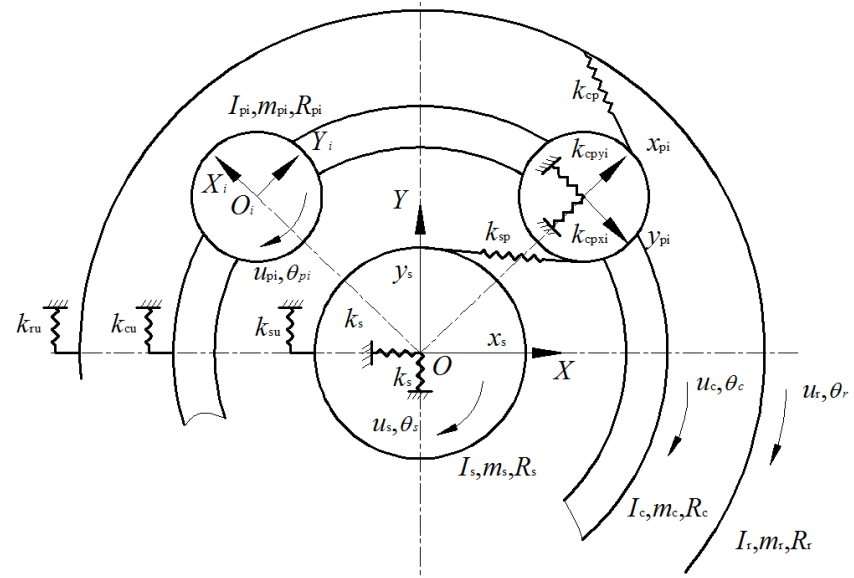

Fig. (2). The dynamic model of the system.

The generalized coupled transverse-torsion dynamic model is established, when the friction on tooth surfaces is neglected. The relative gear mesh displacement of a sunplanet pair can be denoted as:

$\Delta_{s p}=x_{s} \sin \phi_{s p i}-y_{s} \cos \phi_{s p i}+u_{s}-x_{p i} \sin \alpha$

$$
-y_{p i} \cos \alpha-u_{p i}-E_{s p}(t)
$$

The relative gear mesh displacement of a ring-planet pair is given as:

$$
\begin{gathered}
\Delta_{r p}=x_{r} \sin \phi_{r p i}-y_{r} \cos \phi_{r p i}+u_{r}+x_{p i} \sin \alpha \\
-y_{p i} \cos \alpha-u_{p i}-E_{r p}(t)
\end{gathered}
$$

And the relative displacement of carrier-planet is represented as:

$\Delta_{c p x i}=x_{c} \cos \phi_{p i}+y_{c} \sin \phi_{p i}-x_{p i}$

$\Delta_{c p y i}=x_{c} \sin \phi_{p i}-y_{c} \cos \phi_{p i}-y_{p i}+u_{c}$ where, $\alpha$ is the transverse pressure angle, and $\varphi_{s p i}=\alpha+\varphi_{n i}$, $\varphi_{r p i}=\alpha-\varphi_{n i}$, in which $\varphi_{n i}$ is the fixed angle. $E_{s p}$ and $E_{r p}$ are the transmission error of sun-planet and ring-planet pairs respectively. According to Newton's second law, the undamped differential equations of motion of the sun gear can be derived as:

$$
\begin{aligned}
& m_{s} \ddot{x}_{s}+k_{s} x_{s}+\sum_{i=1}^{N} k_{s p} \Delta_{s p i} \sin \phi_{s p i} \\
& =\sum_{i=1}^{N} k_{s p} E_{s p}(t)+m_{s} E_{s} \omega_{s}^{2} \cos \left(\omega_{s} t\right) \\
& m_{s} \ddot{y}_{s}+k_{s} y_{s} \sum_{i=1}^{N} k_{s p} \Delta_{s p i} \cos \phi_{s p i} \\
& =m_{s} g-\sum_{i=1}^{N} k_{s p} E_{s p}(t)+m_{s} E_{s} \omega_{s}^{2} \sin \left(\omega_{s} t\right) \\
& \frac{I_{s}}{R_{s}^{2}} \ddot{u}_{s}+k_{s u} u_{s}+\sum_{i=1}^{N} k_{s p} \Delta_{s p i}=\frac{T_{p}}{R_{s}}+\sum_{i=1}^{N} k_{s p} E_{s p}(t)
\end{aligned}
$$

where, $m_{j}$ and $I_{j}(j=s, r, c, p i, \ldots, p N)$ is the mass and the moment of inertia of each parts, $T_{p}$ and $T_{g}$ denote the input and output torque, respectively. The equations of motion for the ring, carrier and each planet can be obtained similarly.

The differential equations of motion of each planet are given as:

$$
\begin{aligned}
& m_{p} \ddot{x}_{p i}-k_{c p} \Delta_{c p x i}-k_{s p} \Delta_{s p i} \sin \alpha+k_{r p} \Delta_{r p i} \sin \alpha \\
& =m_{p} E_{p} \omega_{p}{ }^{2} \cos \left(\omega_{p} t\right)+m_{p} g \cos \phi_{p i}-k_{s p} E_{s p}(t)+k_{r p} E_{r p}(t) \\
& m_{p} \ddot{y}_{p i}-k_{c p} \Delta_{c p y i}-k_{s p} \Delta_{s p i} \cos \alpha-k_{r p} \Delta_{r p i} \cos \alpha \\
& =m_{p} E_{p} \omega_{p}^{2} \sin \left(\omega_{p} t\right)+m_{p} g \sin \phi_{p i}-k_{s p} E_{s p}(t)-k_{r p} E_{r p}(t) \\
& \frac{I_{p i}}{R_{p}^{2}} \ddot{u}_{p i}-k_{r p} \Delta_{r p i}+k_{s p} \Delta_{s p i} \\
& =m_{p} E_{p} g \cos \left(\omega_{p} t\right)+k_{s p} E_{s p}(t)-k_{r p} E_{r p}(t)
\end{aligned}
$$

For ring gear:

$$
\begin{aligned}
& m_{r} \ddot{x}_{r}+k_{r} x_{r}+\sum_{i=1}^{N} k_{r p} \Delta_{r p i} \sin \phi_{r p i}=\sum_{i=1}^{N} k_{r p} E_{r p}(t)+m_{r} E_{r} \omega_{r}^{2} \cos \left(\omega_{r} t\right) \\
& m_{r} \ddot{y}_{r}+k_{r} y_{r} \quad \sum_{i=1}^{N} k_{r p} \Delta_{r p i} \cos \phi_{r p i}=m_{r} g \sum_{i=1}^{N} k_{r p} E_{r p}(t)+m_{r} E_{r} \omega_{r}^{2} \sin \left(\omega_{r} t\right) \\
& \frac{I_{r}}{R_{r}^{2}} \ddot{u}_{r}+k_{r u} u_{r}+\sum_{i=1}^{N} k_{r p} \Delta_{r p i}=\frac{T_{g}}{R_{r}}+\sum_{i=1}^{N} k_{r p} E_{r p}(t)
\end{aligned}
$$

The differential equations of carrier can be derived as:

$$
\begin{aligned}
& m_{c} \ddot{x}_{c}+k_{c} x_{c}+\sum_{i=1}^{N} k_{c p} \Delta_{c p x i} \cos \phi_{p i} \\
& +\sum_{i=1}^{N} k_{c p} \Delta_{c p y i} \sin \phi_{p i}=m_{c} E_{c} \omega_{c}{ }^{2} \cos \left(\omega_{c} t\right) \\
& m_{c} \ddot{y}_{c}+k_{c} y_{c}+\sum_{i=1}^{N} k_{c p} \Delta_{c p x i} \sin \phi_{p i} \\
& -\sum_{i=1}^{N} k_{c p} \Delta_{c p y i} \cos \phi_{p i}=m_{c} g+m_{c} E_{c} \omega_{c}{ }^{2} \cos \left(\omega_{c} t\right) \\
& \frac{I_{c}}{R_{c}^{2}} \ddot{u}_{c}+k_{c u} u_{c}+\sum_{i=1}^{N} k_{c p} \Delta_{c p y i}=m_{c} E_{c} g \cos \left(\omega_{c} t\right)
\end{aligned}
$$


The system equations can be obtained by assembling the un-damped motion differential equation of each rigid body and derived in matrix form as:

$M \ddot{X}+K X=F$

where, $\boldsymbol{M}$ is the mass matrix, $\boldsymbol{K}$ represents the stiffness matrix, $\boldsymbol{F}$ denotes exciting force vector, and $\boldsymbol{X}$ is the vector of the degrees of freedom of the system.

\section{MODAL ANALYSIS}

The parameters of the investigated PGT are given in Table 1. The planets are assumed identical and equally spaced. For each part, the support have equal stiffness in $x$ and $y$ directions. And all planet support stiffness are equal $\left(k_{c p x i}=k_{\text {cpyi }}=k_{p}\right)$, all sun-planet meshing stiffness $k_{\text {sp }}$ are equal, all ring-planet meshing stiffness $k_{\text {rp }}$ are same. The carrierplanet support stiffness adopts the same value. The sun and ring gear have the same transverse pressure angle.

Table 1. Parameters of the PGT.

\begin{tabular}{|c|c|c|c|c|}
\hline Parameter & Sun & Planet & Ring & Carrier \\
\hline \hline Mass $(\mathrm{kg})$ & 5.0167 & 3.2128 & 20.6353 & 22.4077 \\
\hline Moment of Inertia $\left(\mathrm{kg} \cdot \mathrm{m}^{2}\right)$ & 0.014122 & 0.01689 & 0.60441 & 0.80 \\
\hline Base Radius $(\mathrm{m})$ & 0.05993 & 0.0531 & 0.20117 & 0.15267 \\
\hline Support Stiffness $(\mathrm{N} / \mathrm{m})$ & $7.24 \times 10^{8}$ & $1.56 \times 10^{8}$ & $7.38 \times 10^{9}$ & $1.25 \times 10^{7}$ \\
\hline Torsion Stiffness $(\mathrm{N} / \mathrm{m})$ & 0 & - & $4.63 \times 10^{7}$ & 0 \\
\hline Transverse Pressure Angle $\left({ }^{\circ}\right)$ & \multicolumn{5}{|c|}{$\alpha=28$} \\
\hline
\end{tabular}

The natural frequencies and mode shapes can be calculated by solving the associated eigenvalue problem of equation, which is:

$\omega_{i}^{2} M \phi_{i}=K \phi_{i}$

where, $\omega_{i}$ is the $j$-th natural frequency, $\phi_{i}$ is the corresponding mode shape.

\subsection{Natural Frequencies and Vibration Modes}

By computing the natural characteristics, the different natural frequencies of the PGT system are acquired, and the mode shapes can be classified into three types: translational mode, torsional modes and planetary vibration mode. The natural frequencies and the corresponding vibration modes are shown in Table 2. The system is positive semi-definite and therefore has a rigid body mode, with the frequency of
$0 \mathrm{~Hz}$. For the investigated model, there are six translational modes, five torsional modes and three planetary modes.

In Fig. (3), the normalized modal deflections for the three vibration modes are shown. The planetary vibration mode means that all planets have translational and rotational movements, see Fig. (3a). According to Fig. (3b), the center parts have pure rotational movements, and the translational deflections are zero. For translational mode, see Fig. (3c), the center parts (sun, ring and carrier) have pure translational movements, and the torsional deflections are zero.

\subsection{Influence of Mesh Stiffness on Natural Characteristics}

According to the findings of J. Lin and R. G. Parker, the natural frequency sensitivity to certain stiffness is uniquely associated with the modal strain energy stored in that spring element [3]. The mesh strain energy is generated due to gear distortion, and for the each stage it is defined as:

$U_{j p i}=\frac{1}{2} \sum_{i=1}^{4} k_{m i} \delta_{j p}^{2}(j=s, r)$

where $U_{j p i}$ is the mesh strain energy of each stage for external or internal gearing, $k_{m i}$ is the mean mesh stiffness, $\delta_{s p}$ and $\delta_{r p}$ are the relative deflection as expressed in Eq. (1) to Eq. (3). The derivative of eigenvalue with respect to mesh stiffness $k_{m i}$ is expressed as:

$$
\frac{\partial \lambda_{i}}{\partial k_{m i}}=\delta_{j p}^{2}=\frac{2 U_{j p i}}{k_{m i}}(j=s, r)
$$

Therefore, the mesh strain energy can intuitively reflect the effects of mesh stiffness on the concerned natural frequency. Fig. (4) shows the mesh strain energy of all natural frequencies for external and internal gearing. As the modal deflection has been normalized, the mesh strain energy calculated here is also dimensionless. The $x$-axis is the stage number of each frequency, and $y$-axis is the relative value of mesh strain energy. It can be clearly seen from Fig. (4a) that the mesh stiffness has greater influence on the first, fourth, tenth, eleventh, fourteenth, eighteenth, nineteenth, twentieth, and twenty-first frequencies than other stages. For internal gearing, the effect on the twelfth, thirteenth stages are more obvious, see Fig. (4b).

\subsection{Influence of Support Stiffness on Natural Characteristics}

Similar to the mesh strain energy, the support strain energies for support stiffness are defined as:

Table 2. Natural frequencies and mode types.

\begin{tabular}{|c|c|c|c|c|c|}
\hline Vibration Mode & Frequency & Vibration Mode & Frequency & Vibration Mode & Frequency \\
\hline \multirow{6}{*}{ Torsion } & 535.61 & \multirow{6}{*}{ Translation } & 351.13 & \multirow{6}{*}{ Planetary Vibration } & \multirow{2}{*}{666.28} \\
\hline & 696.92 & & 772.00 & & \\
\hline & 1167.10 & & 1584.21 & & \multirow{2}{*}{4033.64} \\
\hline & 5051.55 & & 2778.36 & & \\
\hline & 6863.23 & & 4738.37 & & \multirow{2}{*}{5148.59} \\
\hline & -- & & 5890.25 & & \\
\hline
\end{tabular}


$\begin{aligned} U_{i} & =U_{i x}+U_{i y}=\frac{1}{2}\left(k_{i x} x_{i}^{2}+k_{i y} y_{i}^{2}\right) \\ (i & =p 1, p 2 \ldots, p 4, s, r, c)\end{aligned}$

where, $U_{i}$ is the total support strain energies in the bearing of gear $i, U_{i x}, U_{i y}$ are the strain energies in the support springs of bearing, respectively. And for planets, $k_{i x}=k_{c p x}, k_{i y}=k_{c p y}$.

(a) Planetary vibration mode

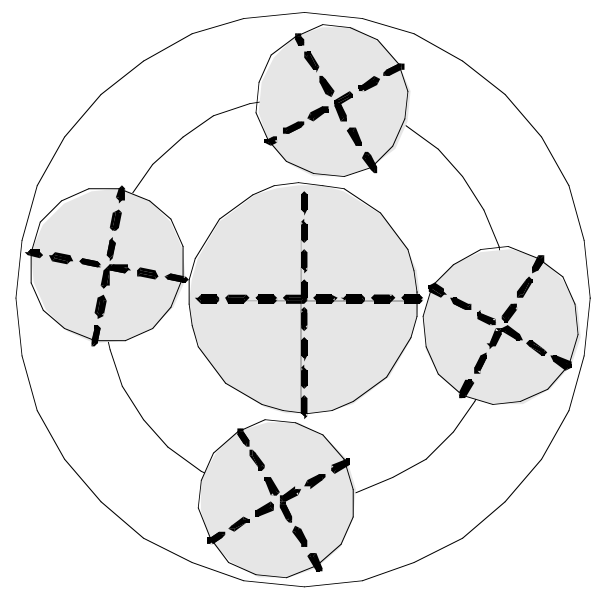

(b) Torsional vibration mode

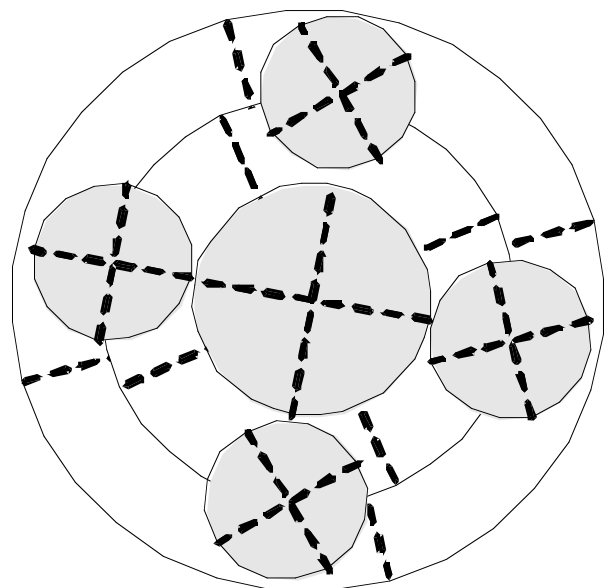

(c) Translational vibration mode

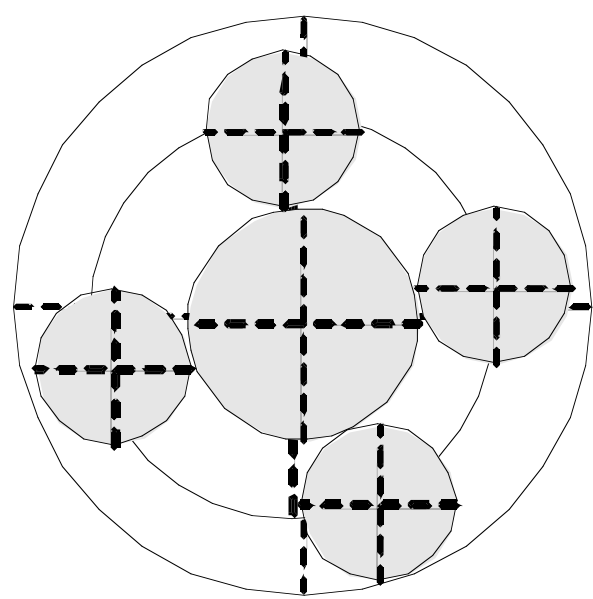

Fig. (3). Modal deflection for the three modes.

(a) Mesh strain energy for external gearing

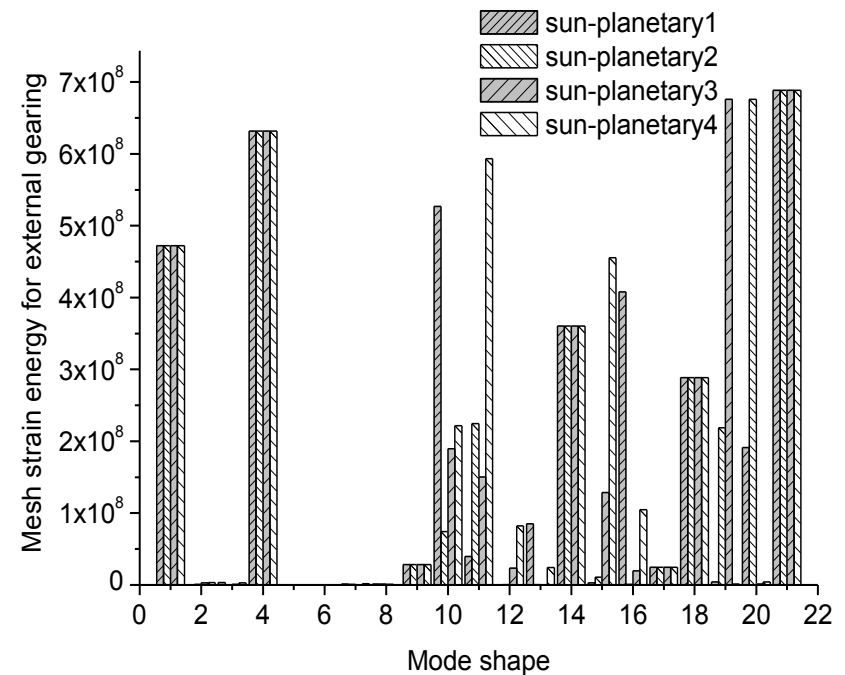

(b) Mesh strain energy for internal gearing

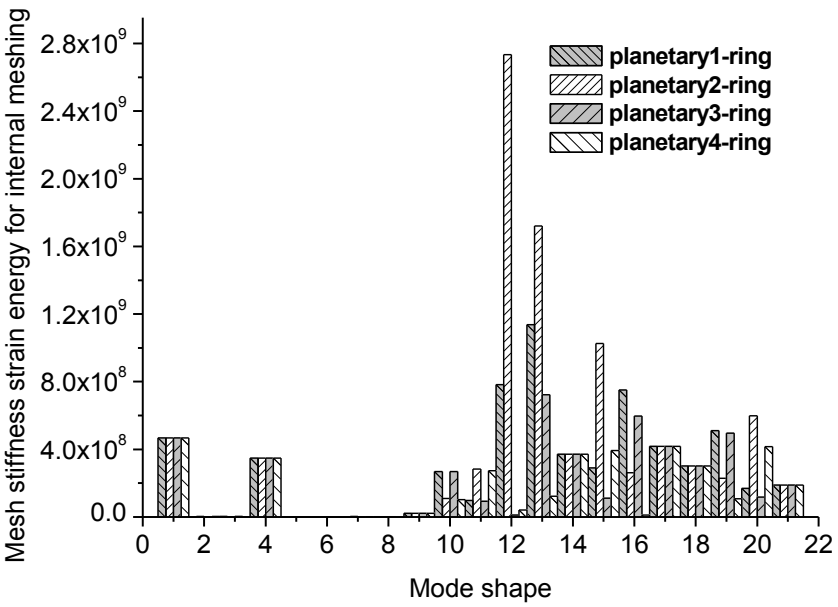

Fig. (4). Mesh strain energy for all mode shapes.

The derivative of eigenvalue with respect to bearing stiffness is:

$\frac{\partial \lambda_{i}}{\partial k_{i}}=2\left(x_{i}^{2}+y_{i}^{2}\right)=4\left(\frac{U_{i x}}{k_{i x}}+\frac{U_{i y}}{k_{i y}}\right)$

Fig. (6) shows the distribution of strain energies for all bearings at $1584.21 \mathrm{~Hz}$ and $4738.37 \mathrm{~Hz}$. As the description in Fig. (5), the $y$ support stiffness of the sun gear is the most sensitive when the natural frequency equals to $1584.21 \mathrm{~Hz}$. And the natural frequency $4738.37 \mathrm{~Hz}$ is most sensitive to the bearing stiffness of the ring gear. It can be also seen that the translational mode $(4738.37 \mathrm{~Hz})$ is almost independent of the bearing stiffness of the carrier because there are very small deflections for them in this mode.

\subsection{Influence of Mass Property on Natural Characteristics}

The modal kinetic energy of the system is defined as:

$U_{i}=\frac{1}{2} \omega_{j}^{2} m_{i}\left(x_{i}^{2}+y_{i}^{2}\right)$ 
$T_{i}=\frac{1}{2} \omega_{j}^{2} m_{e q, i} u_{i}^{2}$

where $U_{i}(i=s, r, c, p)$ is the translational kinetic energy of different gears, $T_{i}(i=s, r, c, p)$ is the kinetic energy associated with a certain moment of inertia.

(a) Supoort strain energy for $1584.21 \mathrm{~Hz}$

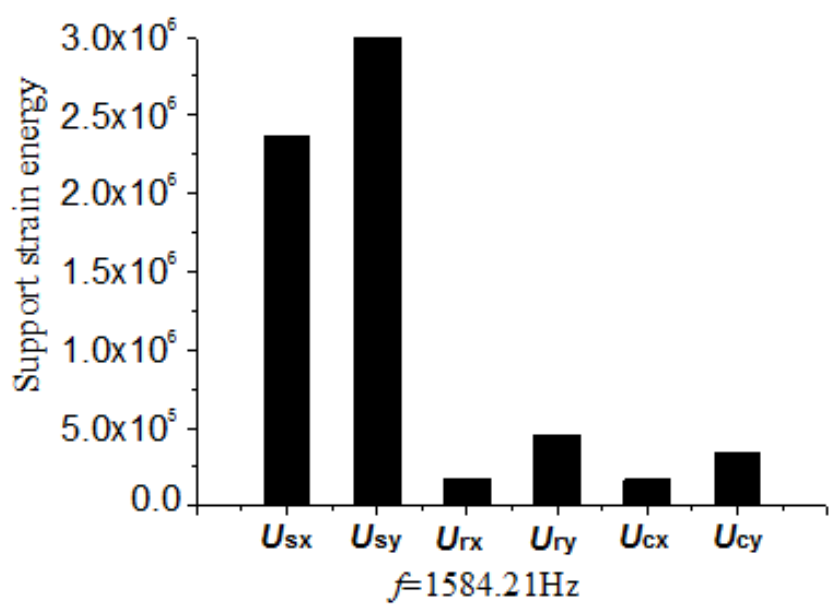

(b) Support strain energy for $4738.37 \mathrm{~Hz}$

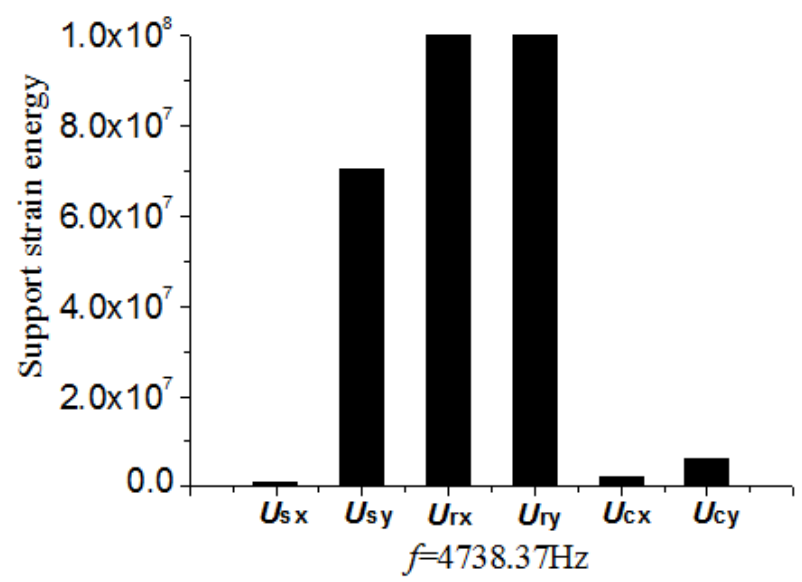

Fig. (5). Support strain energy for different frequencies.

The derivative of eigenvalue with respect to mass is expressed as:

$\frac{\partial \lambda_{j}}{\partial m_{i}}=-\lambda_{j}\left(x_{i}^{2}+y_{i}^{2}\right)=-\frac{2 U_{i}}{m_{i}}$

$\frac{\partial \lambda_{j}}{\partial m_{i}}=-\lambda_{i} u_{i}^{2}=-\frac{2 T_{i}}{m_{e q, i}}$

Therefore, the kinetic energies can be used to estimate the impact of mass properties on different frequencies. Fig. (6) shows the distribution of kinetic energies in $1584.21 \mathrm{~Hz}$ and $6863.23 \mathrm{~Hz}$. As can be seen in Fig. (6a), the moments of inertia of sun and ring have significant effect on the natural frequency $1584.21 \mathrm{~Hz}$. It can be concluded that the torsional deflections of sun and planets may be the principal deflections when the natural frequency equals to $6863.23 \mathrm{~Hz}$. According to the mode shapes of $1584.21 \mathrm{~Hz}$, the torsional deflection of all center gears approaches to zero and the conclusion agrees with Fig. (4c).

(a) Translational mode for $1584.21 \mathrm{~Hz}$

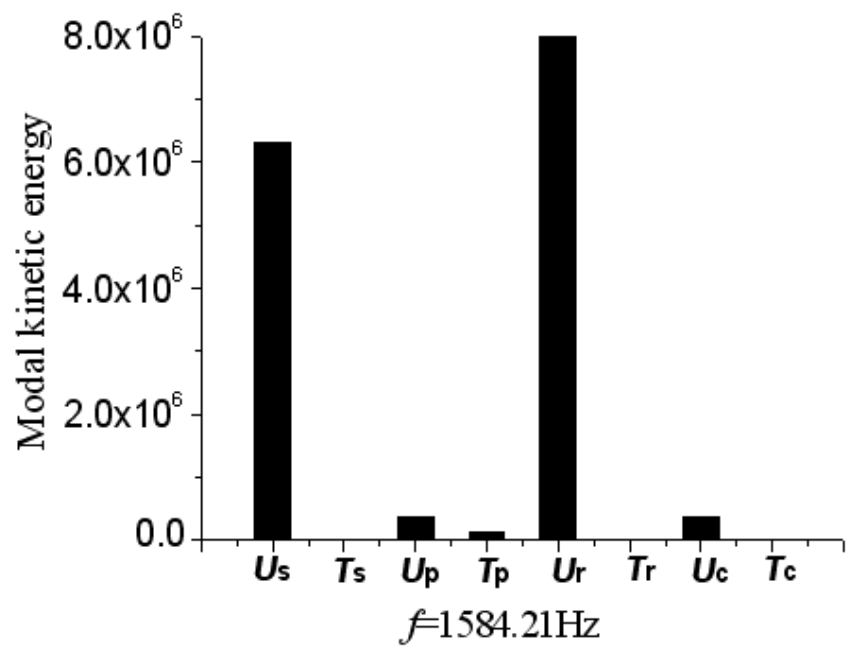

(b) Torsional mode for $6863.23 \mathrm{~Hz}$

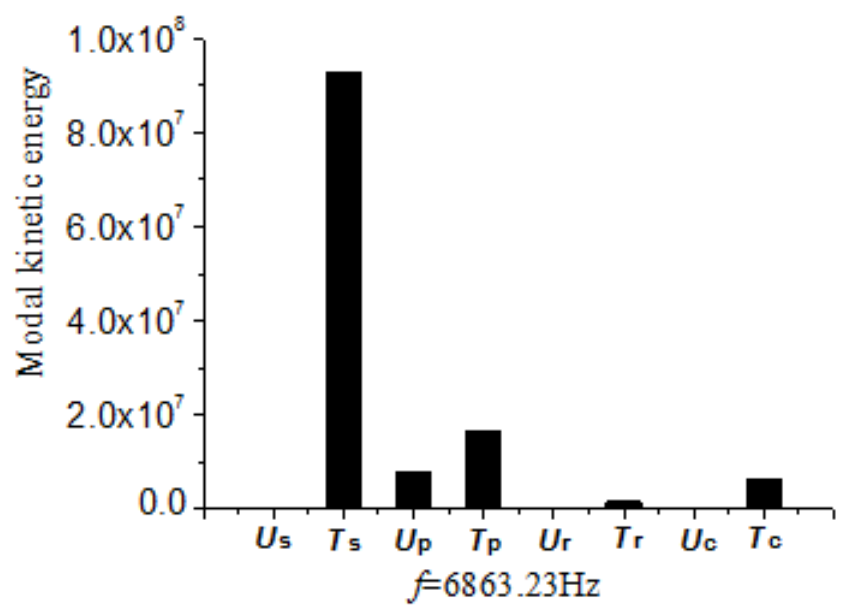

Fig. (6). Distribution of kinetic energies.

\section{CONCLUSION}

A dynamic model of a planetary gear transmission has been developed in order to investigate the free vibration characteristics of the system. The influence of several key design parameters have been discussed in this paper. Three types of vibration modes are acquired, which include translational mode, torsional mode and planetary vibration mode. The simplified formulae for mesh strain energy and support strain energy can directly reflect the influences of mesh stiffness and support stiffness on a certain vibration mode. The support stiffness has no effect on the center gears in torsional mode and the mode shape is independent of the masses of the center wheels, while the translational mode is independent of the moments of inertia of the center gears. The dynamic response and vibration influence of the PGT will become the focus of the future work.

\section{CONFLICT OF INTEREST}

The authors confirm that this article content has no conflict of interest. 


\section{ACKNOWLEDGEMENTS}

This work is supported by the National Natural Science Foundation of China (51205029), the Jiangsu Planned Projects for Postdoctoral Research Funds(1302182C), the Special Fund for Basic Scientific Research of Central Colleges, Chang'an University (CHD2011JC177 and 2013G3252005) and the Engineering Research Center of Expressway Construction \& Maintenance Equipment and Technology (Chang'an University), MOE (2013G1502057).

\section{REFERENCES}

[1] T.L. Krantz, "Mechanical systems technology branch research summary", NASA Technical Memorandum, vol. 10, pp. 63-29, 1993.

[2] D.R. Kiracofe and R.G. Parker, "Structured vibration modes of general compound planetary gear systems", Journal of Vibration and Acoustics, vol. 129, pp.1-16, 2007.

[3] J. Lin and R. G. Parker, "Sensitivity of planetary gear natural frequencies and vibration modes to model parameters", Journal of Sound and Vibration, vol. 228, pp. 109-28, 1999.
[4] J. Lin and, R. G. Parker, "Planetary gear parameteric instability caused by mesh stiffness variation", Journal of Sound and Vibration, vol. 249, pp. 129-45, 2002.

[5] V. K. Ambarisha and R. G. Parker, "Nonlinear dynamics of planetary gears using analytical and finite element models", Journal of Sound and Vibration, vol. 302, pp. 577-95, 2007.

[6] A. Saada and P. Velex, "An extended model for the analysis of the dynamic behaviour of planetary trains", Journal of Mechanical Design, vol.117, pp. 241-7,1995.

[7] A. Kahraman, "Planetary gear train dynamics", Journal of Mechanical Design, vol. 116, pp. 713-20, 1994

[8] A. Fernandez del Rincon, F. Viadero, M. Iglesias, P. García, A. deJuan and R. Sancibrian, "A model for the study of meshing stiffness in spur gear transmissions", Mechanism and Machine Theory vol. 61, pp. 30-58, 2013.

[9] Z. Yang, S.M. Wang, and Y.S. Fan, "Nonlinear dynamics characteristics of split-torque gear transmission system", Chinese Journal of Mechanical Engineering, vol. 44, pp. 52-7, 2008.

[10] M. Rashidi and T. L. Krantz, "Dynamics of a split torque helicopter transmission", NASA Technical Memorandum 105681, 1992.

[11] L.H. Chang, G. Liu, L.Y. Wu and Z.H. Bu, "Research on vibration influence chart of planetary gear systems", Applied Mechanics and Materials, vol. 86, pp. 747-51, 2011.

(C) Zhaoxia et al.; Licensee Bentham Open.

This is an open access article licensed under the terms of the Creative Commons Attribution Non-Commercial License (http://creativecommons.org/licenses/ by-nc/4.0/) which permits unrestricted, non-commercial use, distribution and reproduction in any medium, provided the work is properly cited. 